\title{
ARTICLE OPEN \\ In-situ visualization of hydrogen evolution sites on helium ion treated molybdenum dichalcogenides under reaction conditions
}

\author{
Elmar Mitterreiter (iD) ${ }^{1,2}$, Yunchang Liang ${ }^{3}$, Matthias Golibrzuch², David McLaughlin ${ }^{3}$, Christoph Csoklich ${ }^{3}$, Johannes D. Bartl (iD ${ }^{2,4}$, \\ Alexander Holleitner ${ }^{1,2}$, Ursula Wurstbauer ${ }^{1,2,5}$ and Aliaksandr S. Bandarenka $\mathbb{D}^{1,3,6}$
}

Nanostructured 2D transition metal dichalcogenides play an increasingly important role in heterogeneous catalysis. These materials are abundant (co-)catalysts with tunable properties to catalyze a number of key reactions related to energy provision, for instance the hydrogen evolution reaction (HER). It is vital to understand which surface sites are active in order to maximize their number and to improve the overall (photo-)catalytic behavior of those materials. Here, we visualize these active sites under HER conditions at the surface of molybdenum dichalcogenides $\left(\mathrm{MoX}_{2}, \mathrm{X}=\mathrm{Se}, \mathrm{S}\right)$ with lateral resolution on the nanometer scale by means of electrochemical scanning tunneling microscopy. The edges of single $\mathrm{MoX}_{2}$ flakes show high catalytic activity, whereas their terraces are inactive. We demonstrate how the inert basal planes of these materials can be activated towards the HER with the help of a focused beam of a He-ion microscope. Our findings demonstrate that the He-ion induced defects contribute at lower overpotentials to the HER, while the activity of the edges exceeds the activity of the basal defects for sufficiently high overpotentials. Given the lithographic resolution of the helium ion microscope, our results show the possibility to generate active sites in transition metal dichalcogenides with a spatial resolution below a few nanometers.

npj 2D Materials and Applications (2019)3:25 ; https://doi.org/10.1038/s41699-019-0107-5

\section{INTRODUCTION}

One of the most important challenges for today's world is to reduce fossil fuel consumption in order to protect the environment and to limit the effects of global warming. ${ }^{1,2}$ The use of (photo-)catalytic water splitting and fuel cells provides an excellent concept to enable the conversion between electrical and chemical energy through a hydrogen-based approach. ${ }^{3-6}$ However, typically utilized platinum-group based noble metal catalysts make electrolysis in acidic media expensive. ${ }^{7}$ Transition metal dichalcogenides (TMDCs), such as molybdenum disulfide $\left(\mathrm{MoS}_{2}\right)$ and molybdenum diselenide $\left(\mathrm{MoSe}_{2}\right)$, are reported to be earth abundant and cheaper alternative catalytic materials for the hydrogen evolution reaction (HER). ${ }^{8} \mathrm{MoS}_{2}$ and $\mathrm{MoSe}_{2}$ are twodimensional semiconducting materials with intensive light-matter interactions resulting in an absorption of up to $15 \%$ of the visible solar spectrum even by a single layer. ${ }^{9,10}$ In particular, $\mathrm{MoS}_{2}$ monolayers have a band gap and a band edge alignment appropriate for the water splitting reaction. ${ }^{11,12}$ Compared to bulk $\mathrm{MoS}_{2}$, monolayers, ${ }^{13}$ nanowires, ${ }^{14}$ and vertically aligned molybdenum disulfide thin films ${ }^{15}$ demonstrate an increased catalytic activity regarding the hydrogen evolution reaction (HER).

So far, various forms of $\mathrm{MoS}_{2}$ crystals have been studied with respect to their catalytic activity, ${ }^{16-18}$ starting from physical vapor deposited nanosheets of $\mathrm{MoS}_{2}$ on gold ${ }^{19}$ to $\mathrm{MoS}_{2}$ nanoparticles on graphene oxide, ${ }^{20}$ chemically exfoliated $1 \mathrm{~T} \mathrm{MoS}_{2}$ sheets, ${ }^{21,22}$ chemically synthesized $\mathrm{MoS}_{2}$ nanoparticles, ${ }^{23}$ chemical vapor deposited $\mathrm{MoS}_{2}$ flakes, ${ }^{24}$ large-scale bulk $\mathrm{MoS}_{2}$ monocrystals, ${ }^{25}$ and mechanically exfoliated monolayers of $\operatorname{MoS}_{2} \cdot{ }^{13,26} \mathrm{~A}$ strong increase of the HER was observed with decreasing number of $\mathrm{MoS}_{2}$ layers, suggesting that the cross-plane electron hopping transport across the van-der-Waals gaps in the crystals plays a dominating role in the overall HER process. ${ }^{13,24}$ It is generally understood that the edges of most of the pristine forms of $\mathrm{MoS}_{2}$ exhibit the catalytically dominating sites, whereas the pristine basal planes are typically inactive. ${ }^{20,27}$ In order to activate the basal plane, sulfur vacancies were introduced either during the crystal growth by controlling precursor molecules, ${ }^{23,28}$ by argon and oxygen treatment, ${ }^{29,30}$ as well as by de-sulfurization protocols after growth. ${ }^{31}$ Equally, laser ablation ${ }^{26,32}$ and the implantation of $\mathrm{S}, \mathrm{Se}$ and $\mathrm{Te}$ ions ${ }^{33}$ were reported to increase the basal defect sites and in turn, the HER activity of $\mathrm{MoS}_{2}$. So far, the introduction of sulfur defects has been controlled ex-situ by high-resolution transmission electron microscopy, ${ }^{16,23}$ Raman spectroscopy, ${ }^{16}$ and $x$-ray photoelectron spectroscopy (XPS). ${ }^{28,32}$ Amongst all mentioned forms of $\mathrm{MoS}_{2}$, mechanically exfoliated mono- to few layer $\mathrm{MoS}_{2}$ are regarded to be the best choice for photonic, optoelectronic, and electronic applications, ${ }^{10,34,35}$ but there are only very few studies on their catalytic properties. ${ }^{13,26}$ Scanning tunneling microscopy suggests that mechanically exfoliated monolayers can have a large density of S-vacancies in the basal

\footnotetext{
${ }^{1}$ Nanosystems Initiative Munich (NIM), Schellingstraße 4, 80799 München, Germany; ${ }^{2}$ Walter Schottky Institut and Physik-Department, Technische Universität München, Am Coulombwall 4a, 85748 Garching, Germany; ${ }^{3}$ Physik-Department ECS, Technische Universität München, James-Franck-Str. 1, 85748 Garching, Germany; ${ }^{4}$ Department of

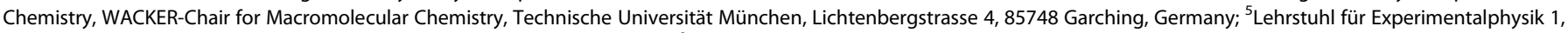
Universität Augsburg, Universitätsstraße 1, 86159 Augsburg, Germany and ${ }^{6}$ Catalysis Research Center TUM, Ernst-Otto-Fischer-Straße 1, 85748 Garching, Germany Correspondence: Alexander Holleitner (Holleitner@wsi.tum.de) or Ursula Wurstbauer (Ursula.Wurstbauer@wsi.tum.de) or Aliaksandr S. Bandarenka (Bandarenka@ph.tum.de) These authors contributed equally: Elmar Mitterreiter, Yunchang Liang.
}

Received: 23 October 2018 Accepted: 20 June 2019

Published online: 15 July 2019 
plane, ${ }^{36-39}$ most likely caused by the exfoliation process. This finding is consistent with a previous report that the HER activity of mechanically exfoliated, pristine monolayers of $\mathrm{MoS}_{2}$ can scale with the area of their basal plane and not with the length of their edges. $^{13}$

In this work, active sites of mechanically exfoliated, few layer $\mathrm{MoS}_{2}$ and $\mathrm{MoSe}_{2}$ are visualized in-situ and in-operando during the hydrogen evolution reaction utilizing an electrochemical scanning tunneling microscope (ECSTM). ${ }^{40}$ This allows us a direct comparison of the HER activity at the edge sites and the basal planes of individual samples. As an initial step towards nanoscale engineering of catalytic active sites for enhanced HER activity, we introduce additional point defects into the basal plane of the few layer $\mathrm{MoS}_{2}$ using the beam of a helium ion microscope (HIM). The high ion energy and a nanometer spot size of the HIM allows to induce defects with high formation energies, such as Mo-vacancies, ${ }^{36}$ in addition to S-vacancies with a low formation energy. ${ }^{19-25,27-32}$ After the helium ion treatment, we demonstrate an overall enhanced activity and visualize in-situ the activated basal plane under HER conditions. All samples are further characterized ex-situ either in vacuum or in the electrolyte using Raman and photoluminescence (PL) spectroscopy, XPS, and optical microscopy. A fundamental understanding of the nature of active sites in molybdenum dichalcogenides as a prototypical semiconducting transition metal dichalcogenides enables further optimization that is expected to result in more efficient devices for electrocatalytic as well as photocatalytic water splitting.

\section{RESULTS AND DISCUSSION}

The study is based on more than 40 different samples consisting of exfoliated $\mathrm{MoS}_{2}$ and $\mathrm{MoSe}_{2}$ flakes on $\mathrm{Au}(111)$ or glassy carbon substrates. In order to get information on the microscopic details of the samples, such as steps and sample edges as well as the flakes' height, we first performed high-resolution STM experiments in ambient conditions. Figure 1 summarizes the STM results on all relevant structures: $\mathrm{MoS}_{2}$ on $\mathrm{Au}(111)(\mathrm{a}-\mathrm{c}), \mathrm{MoS}_{2}$ on glassy carbon (d-f), and $\mathrm{MoSe}_{2}$ on glass carbon (g-i) $\left(\mathrm{MoSe}_{2}\right.$ on $\mathrm{Au}(111)$ is shown in the $\mathrm{SI}$ ). Figure 1a shows the surface of the $\mathrm{Au}(111)$ substrate with atomic resolution. The structure of the surface and the lattice constant are in good agreement with recent literature. ${ }^{41}$ Figure $1 \mathrm{~b}$ depicts a pseudo 3D image of a boundary between $A u(111)$ and $\mathrm{MoS}_{2}$. Open and closed triangles in the figure indicate the step from the substrate to the $\mathrm{MoS}_{2}$ flake and step edges on $\mathrm{MoS}_{2}$, respectively. The basal plane of $\mathrm{MoS}_{2}$ with high resolution is depicted in Fig. 1c. The structure of the basal plane of $\mathrm{MoS}_{2}$ strongly differs from the structure of $A u(111)$ so that one can clearly distinguish between $\mathrm{MoS}_{2}$ and the $\mathrm{Au}(111)$ substrate in
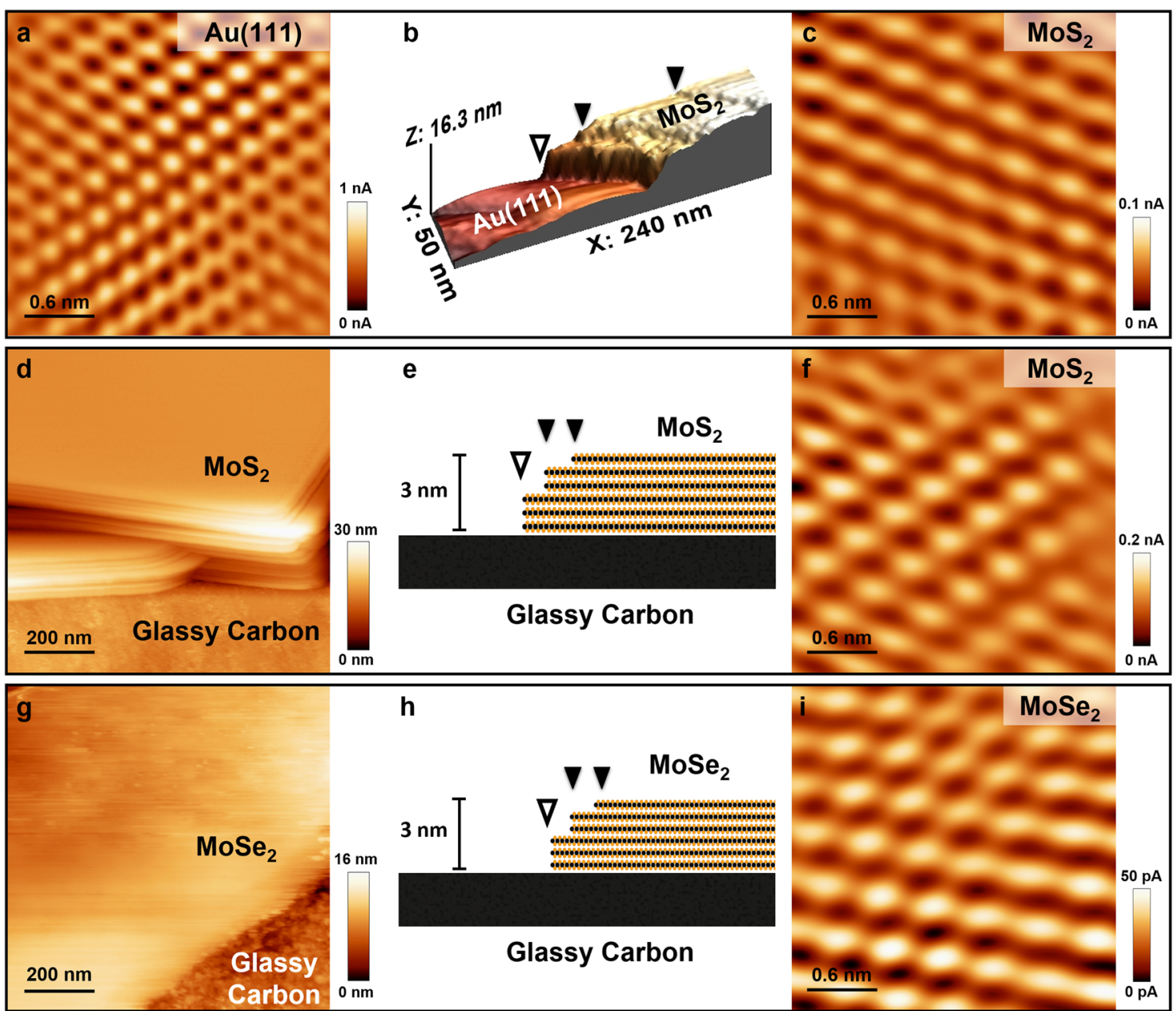

Fig. 1 STM images characterizing $\mathrm{MoS}_{2}$ and $\mathrm{MoSe}_{2}$ flakes on $\mathrm{Au}(111)$ and glassy carbon substrates. a Surface of Au(111) shown with atomic resolution for a corresponding fragment shown in $\mathbf{b}$. $\mathbf{b}$ A boundary between the $\mathrm{Au}(111)$ substrate and a $\mathrm{MoS}_{2}$ flake. This boundary and step edges on $\mathrm{MoS}_{2}$ are highlighted by open and filled triangles. c A basal plane of the surface fragment of MoS shown in $\mathbf{b}$ with high resolution. d $A$ boundary between the glassy carbon substrate and $\mathrm{MoS}_{2}$ with e its schematic structure. $\mathbf{f} A$ fragment of the $\mathrm{MoS}_{2}$ surface shown in $\mathbf{d}$ with high resolution. $\mathbf{g ~ A}$ boundary between the glassy carbon substrate and $\mathrm{MoSe}_{2}$ with $\mathbf{h}$ its schematic structure. i A basal plane of MoSe $\mathrm{S}_{2}$ shown in $\mathbf{g}$ with high resolution 
STM experiments. However, the HER activity of $\mathrm{Au}(111)$ is of the same order of magnitude as the activity of $\mathrm{MoS}_{2}{ }^{42}$ Thus, we also investigated sample structures on glassy carbon as conducting, but catalytically inactive substrate material to reduce the influence of the substrate on the overall activity of the samples.

A pseudo-3D image of the step between the glassy carbon substrate and a MoS 2 flake is shown in Fig. 1d. The surface of $\mathrm{MoS}_{2}$ is atomically smooth, whereas the glassy carbon substrate exhibits a high degree of surface roughness. The layered nature of $\mathrm{MoS}_{2}$ is clearly visible, and the difference between the substrate and the investigated flake is unambiguously evident in the figure. The comparison of several STM images of different $\mathrm{MoS}_{2} /$ glassy carbon boundaries enabled the estimation of the total flake thickness that corresponds to $\sim 30$ layers. Figures $1 \mathrm{e}, \mathrm{f}$ display the schematic structure of the investigated samples and the basal plane of $\mathrm{MoS}_{2}$ with high resolution, respectively. To counter-balance the limited scan range and resolution of our STM measurements at ambient conditions, we utilized a fast Fourier transform (FFT) filter to process the measured STM data in Fig. $1 \mathrm{a}, \mathrm{c}, \mathrm{f}, \mathrm{i}$ (for an asmeasured STM image of $\mathrm{MoS}_{2}$ surface, cf. Supplementary Figure 2). In turn, no defects are visible on the basal planes. However, samples of the same batch show a chalcogenide-vacancy density in the order of $5 \times 10^{12} \mathrm{~cm}^{-2}$ in a high-end STM under vacuum conditions also at room temperature. ${ }^{36}$

As $\mathrm{MoSe}_{2}$ is predicted to be even more active than $\mathrm{MoS}_{2}$ for the $\mathrm{HER}^{40}$ we included this material to our comparative study. Figure $1 \mathrm{~g}-\mathrm{i}$ shows similar measurements as in Fig. $1 \mathrm{~d}-\mathrm{f}$ for $\mathrm{MoSe}_{2}$ on glassy carbon (for STM image of a boundary between a $\mathrm{MoSe}_{2}$ flake and the $\mathrm{Au}(111)$ substrate see Supplementary Fig 1). It should be noted that all investigated systems allow differentiation between the substrate and the TMDC flakes. Importantly, the investigated materials are easy to discern also during the ECSTM measurements. We also characterized the "global" catalytic activity of mechanically exfoliated $\mathrm{MoS}_{2}$ and $\mathrm{MoSe}_{2}$ using linear sweep voltammetry (LSV). Figure $2 a$, b shows the polarization curves on $\mathrm{Au}(111)$ and glassy carbon substrates, respectively. On both substrates, the catalytically activity of $\mathrm{MoSe}_{2}$ exceeds the activity of $\mathrm{MoS}_{2}$. However, we perform the LSV measurements on a huge ensemble of flakes with different thicknesses and sizes. Thus, the LSV such as in Fig. 2 only provides information about the averaged activity of the TMDCs without a distinction between edge and basal sites.

Recently, Pfisterer et al. demonstrated that under reaction conditions additional 'noise' features appear in the ECSTM signal, ${ }^{43}$ which can be directly attributed to the local catalytic activity of different surface sites. When the HER is enabled, additional noise appears close to the active sites of the investigated catalyst. We use this in-situ approach to investigate the active sites at the surface and edges of TMDCs with a high spatial resolution. Figure 3 shows ECSTM images visualizing a few layer $\mathrm{MoS}_{2} / \mathrm{Au}(111)$ boundary (Fig. 3a) as well as a step edge at the surface of a MoS film (Fig. 3b) with both samples immersed in $0.1 \mathrm{M} \mathrm{HClO}_{4}$. The images were taken at different potentials from -600 to $-900 \mathrm{mV}$ versus a platinum pseudo-reference electrode in $50 \mathrm{mV}$ steps. The HER is disabled if the applied potential is $-600 \mathrm{mV}$ or above. Therefore, the images, which correspond to this electrode potential, show only the unperturbed topography of the sample: in Fig. 3a, a sharp edge between $\mathrm{Au}(111)$ and $\mathrm{MoS}_{2}$ is clearly visible for the "HER off" situation. When changing the potential down to $-900 \mathrm{mV}$ corresponding to the "HER on" situation, additional features appear close to the step, and the tunneling current around the edge becomes noisier (Fig. 3a). However, the tunneling current on top of both the $\mathrm{MoS}_{2}$ basal plane and the $A u(111)$ surface stays unaffected. With increasing overpotential, the noise level at the position of the steps becomes more dominant than the current signal determining the height profile and the lateral signature of the edge smears out. A similar behavior is observed for the step edge at the $M_{0} S_{2}$ surfaces shown in Fig. 3b. At a potential of $-900 \mathrm{mV}$ (pseudo-reference electrode), the noise level is the highest and still predominantly located at the step. Figure $3 c$, d shows line profiles taken along the same positions from the scanned area corresponding to different overpotentials. The black arrows in Fig. 3c, d indicate increasing overpotentials, i.e., acceleration of the HER. From the analysis of Fig. 3, it is evident that the most active HER sites are located close to the edges and the steps, as the noise appears over the lateral positions with the highest electrocatalytic activity. ${ }^{43}$ The gold substrate shows no activity in the investigated potential range, which is in good agreement with literature. ${ }^{44-46}$ No catalytic activity is visible also on the basal planes of the $\mathrm{MoS}_{2}$ sample without steps.

Mechanically exfoliated, few layer $\mathrm{MoS}_{2}$ samples are reported to have a significant amount of point defects in the lattice $\left(\sim 5 \times 10^{12}\right.$ $\left.\mathrm{cm}^{-2}\right) .36,37$ The point defects are predominantly sulfur vacancies $^{38,39}$ that are supposed to be catalytically active for the HER. ${ }^{47}$ For monolayers of mechanically exfoliated $\mathrm{MoS}_{2}$, it was recently deduced that not only the active edge sites but also most likely such point defects contribute to the overall HER activity. ${ }^{13}$ For this reason, it is somewhat surprising that we do not observe signatures for active sites in the basal planes of the investigated few layer $\mathrm{MoS}_{2}$. However, sulfur vacancies in $\mathrm{MoS}_{2}$ are reported to be very mobile and tend to diffuse into the bulk material. ${ }^{48,49}$ This self-healing of defects should significantly reduce the defect density on the surface. ${ }^{39}$ Based on the ECSTM-measurements, we conclude that the edge sites are the dominant active sites in few layer $\mathrm{MoS}_{2}$. ECSTM-measurements on monolayers are beyond the current work because of the technical and temporal challenge to find single monolayers on a substrate with this rather local technique.

Next, we performed similar in-situ ECSTM measurements on few layer $\mathrm{MoSe}_{2}$ flakes deposited on $\mathrm{Au}(111)$ substrates. For $\mathrm{MoSe}_{2}$, up to now there is only little knowledge about the nature of active sites and their detailed behavior under the HER conditions. Figure 4 shows ECSTM images, similar to those depicted in Fig. 3, for a major step edge with several minor steps at the surface of the $\mathrm{MoSe}_{2}$ film. Figure 4a summarizes the set of ECSTM experiments at different applied potentials from -500 to $-800 \mathrm{mV}$ in $50 \mathrm{mV}$ steps, with line profiles being displayed in Fig. 4b. The overall observed behavior of $\mathrm{MoSe}_{2}$ is very similar to that for $\mathrm{MoS}_{2}$. At a low overpotential, neither the surface nor the steps show significant noise features of the tunneling current. An increased noise level and hence HER activity appears at the step and defect sites at a potential of about $-600 \mathrm{mV}$, and the activity at both site locations increases for a higher overpotential. Again, the $\mathrm{Au}(111)$ substrate and the basal plane of $\mathrm{MoSe}_{2}$ display almost no significant noise features in the tunneling current and hence no HER activity. Within the error of the potential of the platinum pseudo reference electrode, the onset potentials found for $\mathrm{MoS}_{2}$ and $\mathrm{MoSe}_{2}$ are in good agreement and corroborate theoretical predictions ${ }^{40,50}$ and initial experimental results. ${ }^{51}$

The natural question arises whether it is possible to create additional active sites on the basal planes of the investigated TMDCs to increase the overall activity. Therefore, we focus on intentionally prepared, defective TMDC surfaces. In order to introduce lattice defects to the basal planes, we bombarded large areas of $\mathrm{MoS}_{2}$ samples with accelerated He-ions (30 keV). The coverage of $\mathrm{MoS}_{2}$ on $\mathrm{Au}(111)$ and glassy carbon electrodes was approximately 20 to $30 \%$. We applied helium ion doses up to 14 ions $/ \mathrm{nm}^{2}$ (56 ions $/ \mathrm{nm}^{2}$ ) for the $\mathrm{MoS}_{2}$ flakes immobilized on $\mathrm{Au}$ (111) (glassy carbon). Figure 5a shows the macroscopic current density vs the overpotential measured by linear sweep voltammetry using a rotating disc electrode. The current density is a measure for the HER activity integrated over all active sites on a sample. Figure $5 a$ displays the current densities for the untreated (blue) and He-ion treated (red) few layer $\mathrm{MoS}_{2}$ on $\mathrm{Au}(111)$ (top panel) and glassy carbon (bottom panel) substrates. All current 

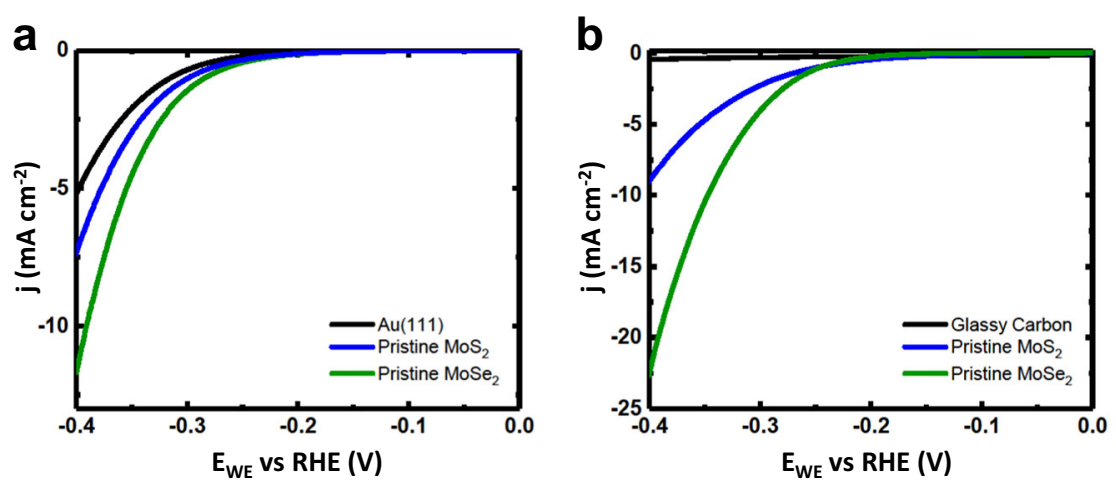

Fig. 2 Catalytic activity measurements of bulk $\mathrm{MoS}_{2}$ and $\mathrm{MoSe}_{2}$ on $\mathrm{Au}(111)$ and glassy carbon. a Linear sweep voltammograms of Au(111), $\mathrm{MoS}_{2}$ on $\mathrm{Au}(111)$ and $\mathrm{MoSe}_{2}$ on $\mathrm{Au}(111)$ in black, blue and green, respectively. $\mathbf{b}$ Linear sweep voltammograms of glassy carbon, MoS ${ }_{2}$ on glassy carbon and $\mathrm{MoSe}_{2}$ on glassy carbon in black, blue and green, respectively
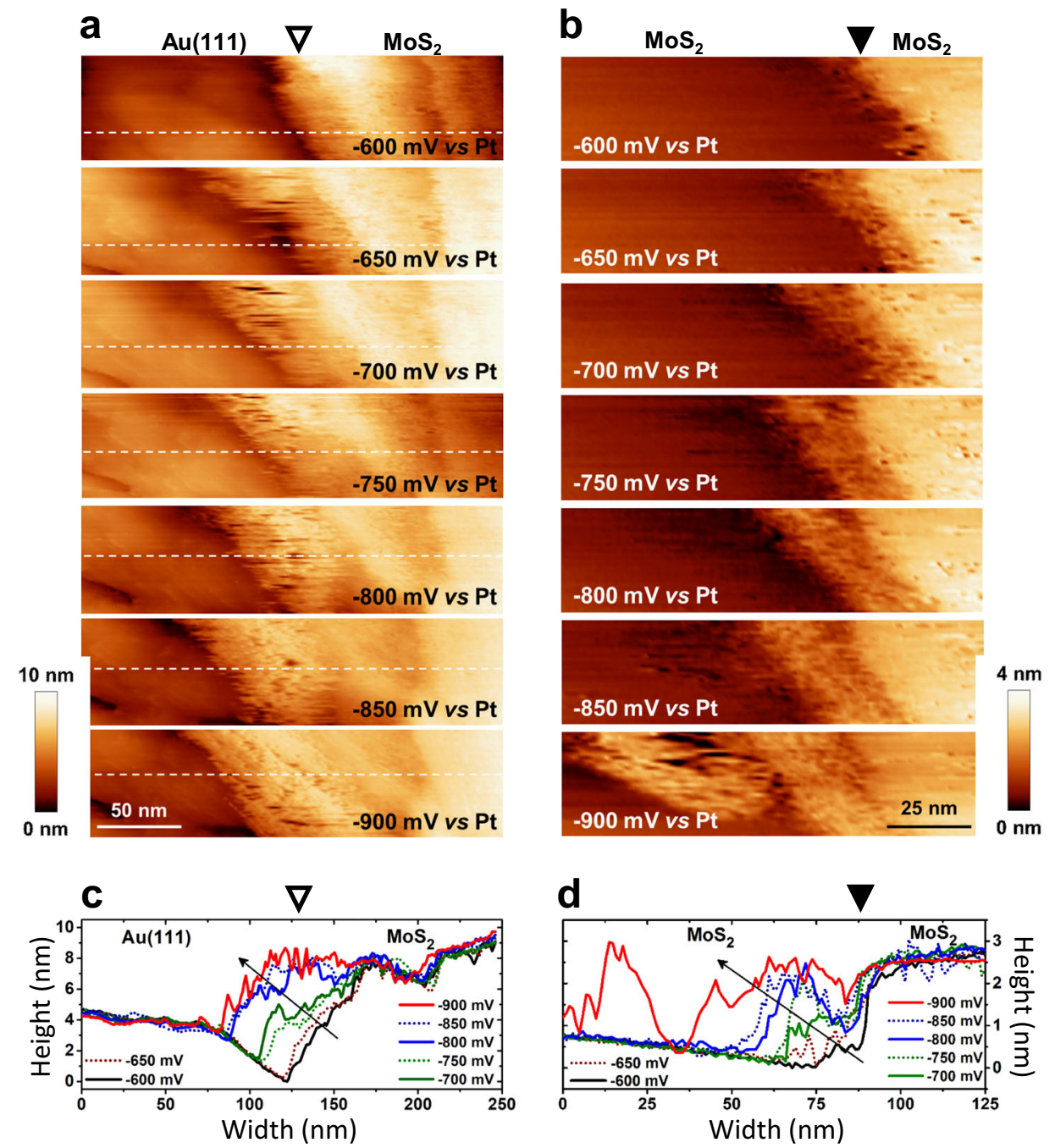

Fig. 3 Constant current mode electrochemical STM images visualizing a $\mathrm{MoS}_{2} / \mathrm{Au}(111)$ boundary and a step edge at the surface of the $\mathrm{MoS}_{2}$ film under HER conditions with increasing overpotentials. a, b A set of images characterizing the a MoS $\mathrm{M}_{2} / \mathrm{Au}(111)$ boundary (open triangle) and $\mathbf{b}$ a step edge at the surface of the $\mathrm{MoS}_{2}$ film (filled triangle) at different electrode potentials as indicated in the figure. $\mathbf{c}$, $\mathbf{d}$ Line profiles across the same position for each potential from $\mathbf{a}$ and $\mathbf{b}$, respectively. In $\mathbf{a}$, the white dashed lines indicate the location where the profile lines were taken. In $\mathbf{b}$, the topmost lines were taken as the drift was minimized during scanning. A pseudo-3D representation of the ECSTM images in $\mathbf{a}, \mathbf{b}$ are shown in supplementary data Figs 3 and 4 , respectively 

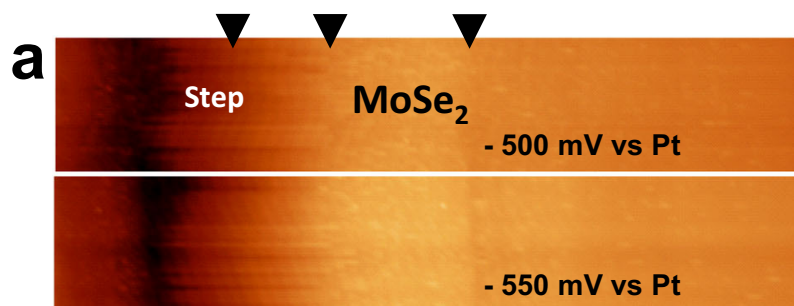

$600 \mathrm{mV}$ vs Pt
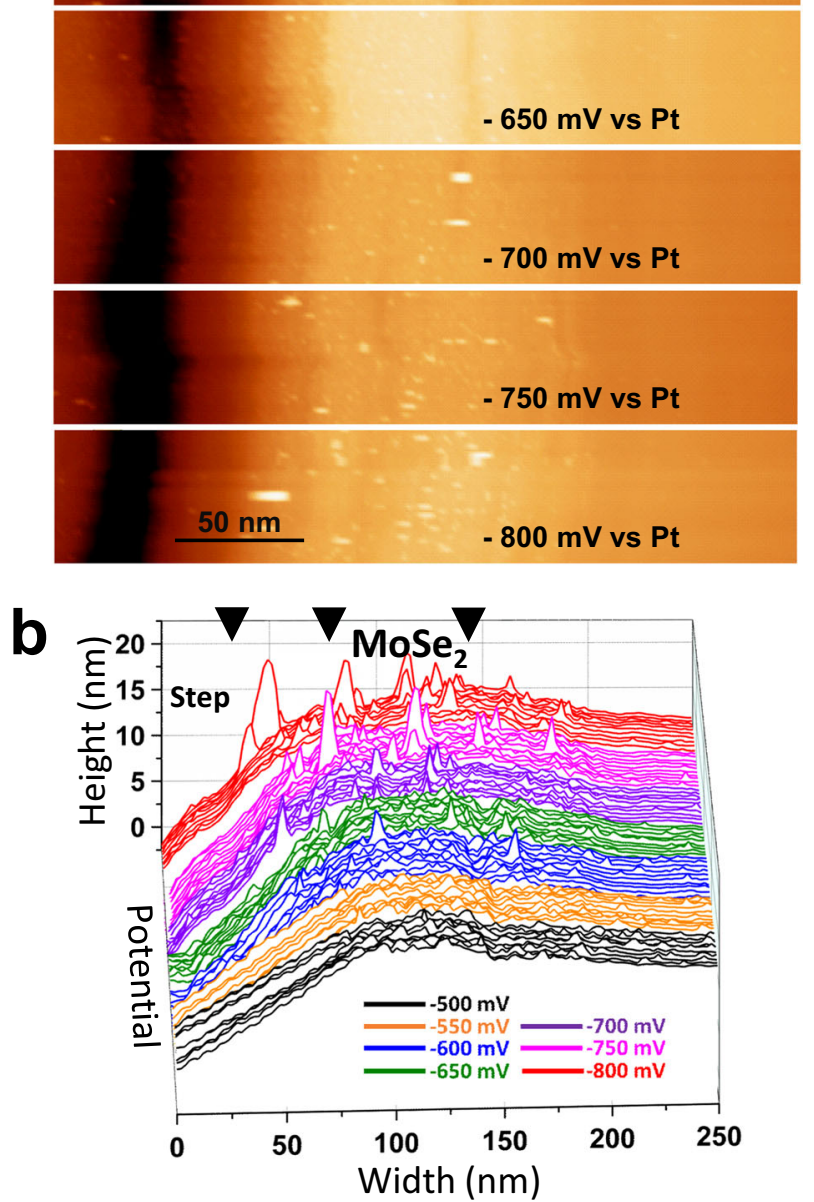

Fig. 4 Constant current mode electrochemical STM images visualizing a major step edge and step-like defects at the surface of the $\mathrm{MoSe}_{2}$ film on $\mathrm{Au}(111)$ under HER conditions with increasing overpotentials. a A set of images visualizing a major step edge and minor steps (filled triangles) at the surface of the $\mathrm{MoSe}_{2}$ film at different electrode potentials as indicated in the figure. b Comparison of line profiles for each potential from a

densities are normalized to the geometric electrode surface area. The HER activities of the substrates are in good agreement with those reported in the literature. ${ }^{13,52}$ After the HIM treatment, the current densities and, hence, the HER activities increased for both samples almost by a factor of 2 (Fig. 5a). The bare substrates without $\mathrm{MoS}_{2}$ did not show any increased activity after the HIM treatment (Supplementary Fig 6). Consequently, the enhanced HER activity found for the He-ion treated $\mathrm{MoS}_{2}$ is most likely induced by the additional defects located at the basal planes. In order to visualize the catalytic activity of the basal planes, we performed ECSTM experiments on the He-ion treated $\mathrm{MoS}_{2}$ samples. In Fig. 5b-d, we compare ECSTM images for untreated and $\mathrm{He}$ ion-treated $\mathrm{MoS}_{2}$ flakes immobilized at the $\mathrm{Au}(111)$ substrates. Figure $5 \mathrm{~b}$ shows the results of the measurements using the untreated $\mathrm{MoS}_{2}$ samples under the "HER on" (potential of $-800 \mathrm{mV}$ vs Pt) conditions. Again, as demonstrated in Fig. 3 and Fig. 4, only the edges of the few layer TMDC flakes are active under the HER reaction conditions as indicated by the corresponding enlarged noise level. The open triangles in the inset of Fig. $5 \mathrm{~b}$ indicate the regions with an increased noise level at the flake edges. Figures 5c, d compare the ECSTM data for different electrode potentials corresponding to the "HER off" ( $-600 \mathrm{mV}$ vs $\mathrm{Pt})$ and "HER on" ( $-800 \mathrm{mV}$ vs Pt) conditions, respectively. The Heion treated surface of $\mathrm{MoS}_{2}$ appears smooth. Neither the basal plane nor the edges display signatures of an increased noise in the tunneling current, when the electrode potential is not negative enough to start the hydrogen evolution reaction. When the electrode potential is negative enough to enable the HER, there is a significant noise in the measured tunneling current at the edges and on the basal plane of the He ion-treated $\mathrm{MoS}_{2}$ flake, but not over the $A u(111)$ substrate. The increased noise level across the whole $\mathrm{MoS}_{2}$ flake is interpreted to signify that the whole basal plane is activated for the HER by the HIM treatment. The magnitude of the measured noise strongly depends on the potential applied to the sample.

Figure 6 shows detailed in-situ line scans taken from a series of ECSTM images at the boundary of the $\mathrm{Au}(111)$ substrate and a HIM treated $\mathrm{MoS}_{2}$ flake. Three different potentials $-600 \mathrm{mV}$ vs Pt ("HER off"), $-700 \mathrm{mV}$ vs Pt ("HER on") and $-800 \mathrm{mV}$ vs Pt ("HER on") are shown in black, blue and red, respectively. At $-600 \mathrm{mV}$ vs $\mathrm{Pt}$, the HER is disabled and the line profiles show almost no noise (black). In turn, the topography of the boundary can be seen. For low overpotentials ( $-700 \mathrm{mV}$ vs Pt, blue curves), the basal plane shows noise features, whereas the edge remains unaffected. The He-ion treated $\mathrm{Au}(111)$ surface shows no HER activity. For more negative electrode potentials $(-800 \mathrm{mV}$ vs $\mathrm{Pt}$, red) also the edge sites become noisy. The noise level at the edge sites is higher than that over the induced crystal defects in the basal plane. This observation suggests the presence of two different types of active sites with different energetics. The defect sites in the basal plane contribute to the HER already at low overpotentials, when the edges are still not active. This suggests, that the energetics of defect sites is favorable for the HER compared to the energetics of the edges. However, for higher overpotentials, when also the edges contribute to the reaction, the HER activity from the $\mathrm{MoS}_{2}$ edges is superior compared to the HER activity from the basal planes. Such a behavior is in good agreement with the predicted hydrogen adsorption free energy of $0.06 \mathrm{eV}$ for edge sites and $0.18 \mathrm{eV}$ for sulfur vacancies at the used density obtained from theoretical studies. ${ }^{53,54}$ The overall increased current densities found for the He ion-treated $\mathrm{MoS}_{2}$ samples from the integrated LSV (Fig. 5a) are in good agreement with the HER activation of the basal plane found in the spatially resolved ECSTM measurements. According to theoretical studies, the hydrogen adsorption free energy $\Delta G_{\mathrm{H}}$ at the basal plane of $\mathrm{MoS}_{2}$ is lowered from $\sim 2.1$ to $0.2 \mathrm{eV}$ at a defect density of $3 \%{ }^{54}$ Its minimum is predicted for a defect density of around $14 \%$. These values are still higher than the adsorption free energy of $\mathrm{MoS}_{2}$ edge sites of $0.06 \mathrm{eV} .^{53,54}$ The adsorption free energy $\Delta G_{H}$ should be as close to zero as possible, to maximize the catalytic activity of $\mathrm{MoS}_{2}$ for the hydrogen evolution reaction. Hence, additional defect engineering can further increase the activity of $\mathrm{MoS}_{2}$.

Raman spectroscopy allows us to determine an effective interdefect distance of the helium ion induced crystal defects to be approximately $2 \mathrm{~nm}$ before the ECSTM measurements (cf. supplementary Fig 7). Such an effective inter-defect distance corresponds to a defect density of about $\sim 3 \cdot 10^{13} \mathrm{~cm}^{-2}$ slightly exceeding the reported density of sulfur vacancies of $\sim 5 \cdot 10^{12}$ $\mathrm{cm}^{-2}$ in pristine samples. ${ }^{36}$ In principle, this nominally increased 
a

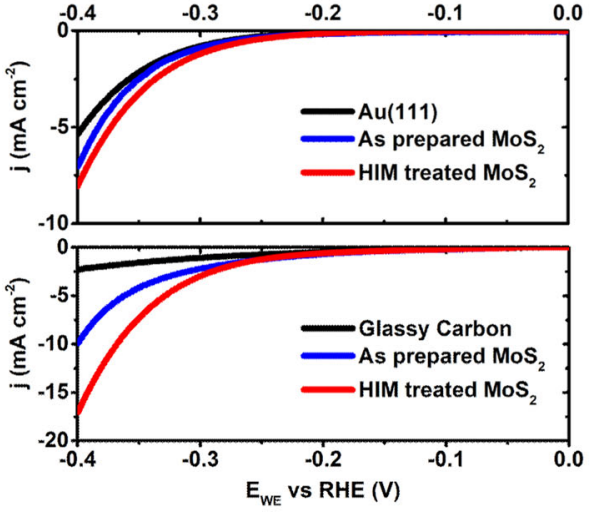

C

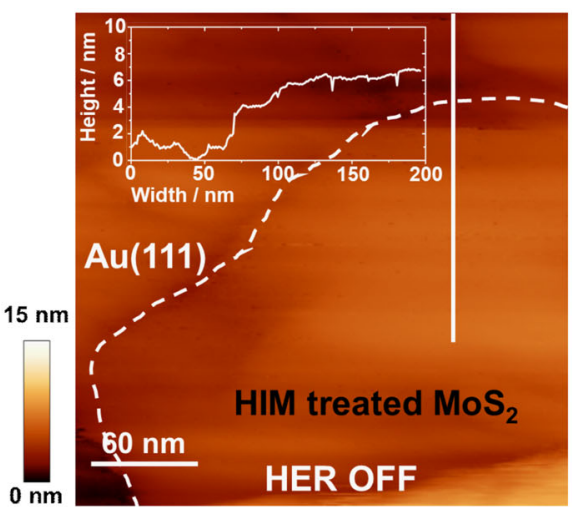

b

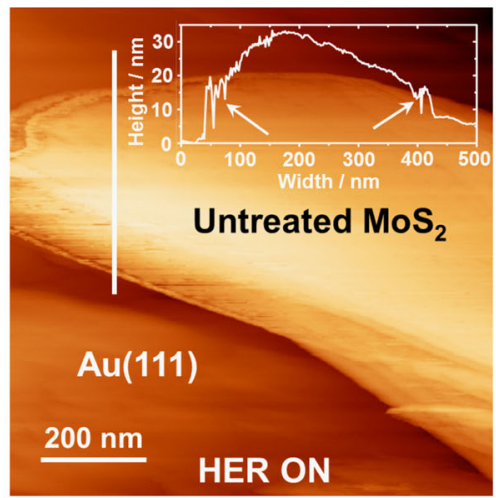

d

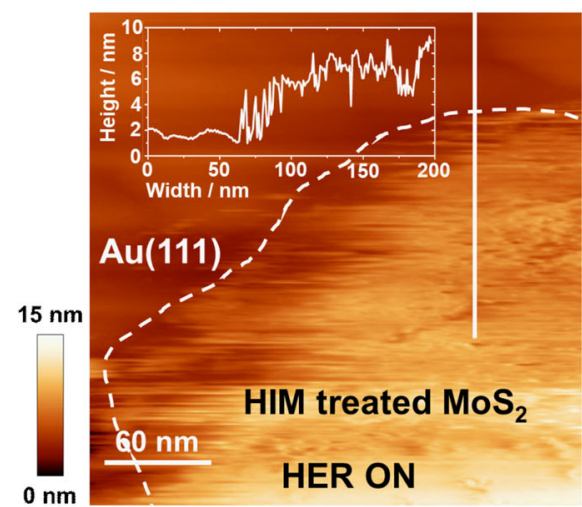

Fig. 5 Catalytic activity of untreated and He ion-treated $\mathrm{MoS}_{2}$ surfaces. a Macroscopic HER-activities of untreated and He ion-treated MoS flakes on $\mathrm{Au}(111)$ (top panel) and on glassy carbon (bottom panel) measured using a rotating disc electrode configuration. $\mathbf{b}$ ECSTM image of untreated $\mathrm{MoS}_{2}$ sample under reaction conditions ( $-800 \mathrm{mV}$, "HER on"): only the boundaries of MoS and Au(111) are active (open triangles in the inset highlighting the steps along a line profile taken along the bottom x-axis of the inset). $\mathbf{c}$, $\mathbf{d}$ ECSTM images of He ion-treated MoS ${ }_{2}$ for the condition c when HER is not possible $(-600 \mathrm{mV}$, "HER off") and $\mathbf{d}$ when HER is enabled ( $-800 \mathrm{mV}$, "HER on"). For a potential low enough to enable HER (same value as in panel b), the whole $\mathrm{He}$ ion-treated $\mathrm{MoS}_{2}$ surface contributes to the HER activity identified from the homogeneous spread of the noise level. The insets in $\mathbf{b}$ - $\mathbf{d}$ display the line profile along the bottom $\mathbf{x}$-axis of the inset indicating the noise level in the different spatial regions at each measurement

density explains why the basal plane appears to be activated in the ECSTM-measurement after the HIM treatment. ${ }^{39,48,49}$ However, the mobility of sulfur vacancies in bulk $\mathrm{MoS}_{2}$ in contact with electrolytes is known to be relatively high ${ }^{39,48,49}$; thus, we probably overestimate the S-vacancy density in-operando. An alternative explanation of the basal activity is the generation of defects with a larger formation energy, such as Mo-vacancies and defect complexes, in the basal plane, as a recent photoluminescence study suggests for monolayer $\mathrm{MoS}_{2}{ }^{55}$ The Mo-vacancies are less mobile than S-vacancies, such that they can additionally contribute to the HER. We point that we do not observe a shift in the sulfur $2 \mathrm{p}$ core region at $162 \mathrm{eV}$ after the defect engineering (cf. Supplementary Figure 10) in contrast to refs. ${ }^{28,29,32}$, where this observable was taken as an ex-situ measure for the presence of Svacancies. Moreover, in our XPS data, the stoichiometric ratio Mo/ $S$ is constant before and after the helium treatment (cf. Supplementary Table 1). In this respect, the presented in-situ ECSTM characterization of the catalytically active sites seems to be more sensitive than the standardized ex-situ XPS method for the investigated samples.

In conclusion, we visualized catalytically active sites in few layer $\mathrm{MoS}_{2}$ and $\mathrm{MoSe}_{2}$ in-situ and in-operando under the HER conditions with high spatial resolution. For the unmodified few layer 2D materials, the most active catalytic centers are located at the boundaries (edge sites) and step sites. In contrast, we observed almost no catalytic activity at the basal planes. We could successfully generate HER-active defects at the surface of $\mathrm{MoS}_{2}$ with the help of a helium ion microscope. The overall catalytic activity of the $\mathrm{MoS}_{2}$ flakes was almost doubled already at low defect densities (with an estimated minimum $2 \mathrm{~nm}$ inter-defect distance before catalytic operation). Our results indicate that the defects introduced by the helium ion beam can not only activate the $\mathrm{MoS}_{2}$ basal plane, but they also lower the required HER overpotential. At sufficiently negative potentials, the HER activity of edge sites exceeds the activity of the defects in the basal plane. We assume that the helium treatment introduces catalytically active chalcogenide mono-vacancies as well as less mobile defects with a larger formation energy, such molybdenum vacancies and defect complexes. Since the number of active edge sites in natural $\mathrm{MoS}_{2}$ and $\mathrm{MoSe}_{2}$ is limited, the introduction of active sites in the basal plane provides a powerful tool to increase the activity of molybdenum dichalcogenides towards the hydrogen evolution reaction.

\section{METHODS}

\section{Sample preparation}

We investigate two different material systems: first, single layer $\mathrm{MoS}_{2}$ and $\mathrm{MoSe}_{2}$ for all fundamental pre-characterizations; second, ensembles of $\mathrm{MoS}_{2}$ and $\mathrm{MoSe}_{2}$ thin films for the electrochemical STM and catalytic 


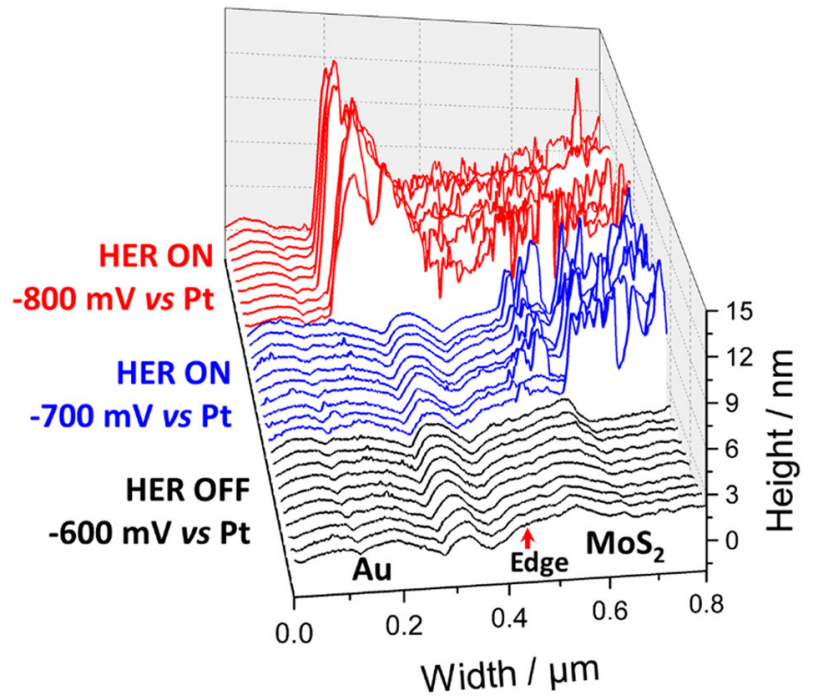

Fig. 6 Potential dependent HER activity of helium ion treated $\mathrm{MoS}_{2}$. Line scans taken from a series of constant current mode ECSTM images of a boundary of a HIM treated $\mathrm{MoS}_{2}$ flake and the $\mathrm{Au}(111)$ substrate in $0.1 \mathrm{M} \mathrm{HClO}_{4}$. The ECSTM images were captured over approximately the same area at the $\mathrm{MoS}_{2} / \mathrm{Au}(111)$ boundary at $-600 \mathrm{mV}$ vs Pt ("HER off"), $-700 \mathrm{mV}$ vs Pt ("HER on") and $-800 \mathrm{mV}$ vs Pt ("HER on"), respectively. Current setpoint: $0.5 \mathrm{nA}$, tip potential: $-100 \mathrm{mV}$ vs Pt, scan rate: $0.5 \mathrm{~Hz}$. The red arrow indicates the location of the $\mathrm{MoS}_{2} / \mathrm{Au}$ boundary

activity measurements. TMD flakes are micromechanically exfoliated from bulk crystals (SPI Supplies) using adhesive tape. Single layer flakes are transferred to a substrate with micrometer precision, following an all-dry viscoelastic stamping technique adapted from ref. ${ }^{56}$ using polydimethylsiloxane (PDMS). In order to increase the transfer probability and to enhance the electrical contact to the substrate, we heat the substrate to approximately $100{ }^{\circ} \mathrm{C}$ during the transfer..$^{57}$ Directly after the transfer, the samples are carefully cleaned using acetone, isopropanol and nitrogen gas, to remove PDMS residues and dirt. A similar procedure is also used to fabricate multilayer TMD samples with high coverages. After the exfoliation steps, the TMD flakes are directly transferred from the adhesive tape. Again, the substrate is heated to approximately $100^{\circ} \mathrm{C}$ during the transfer. Thus, we reach a TMD coverage of the substrate up to $50 \%$. The same cleaning steps as for single layer flakes are processed.

We used three different materials as substrates. First, $3 \mathrm{~mm}$ thick glassy carbon (Hochtemperatur Werkstoffe GmbH SIGRADUR ${ }^{\circ} \mathrm{G}$ ) substrates were polished using diamond powder for macroscopic HER activity measurements and STM investigations. Second, we used Au coated glass chips $\left(11 \times 11 \mathrm{~mm}\right.$, Arrandee $\left.{ }^{\mathrm{TM}}\right)$ as substrates for STM and ECSTM investigations. Finally, for macroscopic HER activity measurements on $\mathrm{Au}(111)$ substrates, we used a bead-type $\mathrm{Au}(111)$ (Icryst, Jülich Germany) single crystal. All Au substrates were flame-annealed using an isobutene flame and cooled down to room temperature in order to have well-defined (111)-dominating surfaces. ${ }^{58}$ We cleaned all substrates directly before the TMD flakes transfer with acetone, isopropanol and nitrogen gas.

\section{Characterization techniques}

Raman/ Photoluminescence spectroscopy. We used optical characterization methods including $\mu$-Raman and photoluminescence (PL) measurements, to gain information about the substrates and TMD flakes. A detailed description of the homemade microscope set-up is summarized in ref. ${ }^{57}$ The $488 \mathrm{~nm}$ laser line of a $\mathrm{Kr} / \mathrm{Ar}$-ion gas laser was focused using a 100x objective (Zeiss LD EC Epiplan-Neofluar 100x/0,75 DIC M27). The typical laser spot size is about $1 \mu \mathrm{m}$ on the sample. The scattered and emitted light was then analyzed in a $500 \mathrm{~mm}$ spectrometer (Princeton Instruments) and was dispersed by 1800 grooves $/ \mathrm{mm}$ ( $\mu$-Raman) or 300 grooves $/ \mathrm{mm}$ $(\mathrm{PL})$. The laser power on the sample $\mathrm{P}=0.1 \mathrm{~mW}$ in all measurements.

Helium ion microscope. We use a helium ion microscope (HIM) to nanostructure the $\mathrm{MoS}_{2}$ flakes on glassy carbon and $\mathrm{Au}(111)$ supporting substrates (HIM ORION NanoFab). A detailed description of the HIM was summarized by Hlawacek. ${ }^{59}$ In order to induce defects into a large area of the sample surface (up to $2 \mathrm{~mm}^{2}$ ), we use a defocused helium ion beam This method ensures an equally introduction of defects into the exposed area. The helium ion dose $D_{H_{e}}$ is calculated using the beam current $I_{b}$, the exposed area $A$, the exposure time $t_{e}$ and the elementary charge $e$.

$D_{H e}=\frac{I_{b} t_{e}}{A e}$

We use high beam currents up to $180 \mathrm{pA}$ at a helium pressure of $3 \cdot 10^{-6}$ Torr and a beam energy of $30 \mathrm{keV}$ to obtain defect densities up to $8 \cdot 10^{13} \mathrm{~cm}^{-2}$. We calculate the defect density assuming that every sulfur vacancy is created in the upper most layer of $\mathrm{MoS}_{2}$. The defect density in a hexagonal lattice can be calculated using the following expression:

$\rho=\frac{2}{\sqrt{3} L_{D}}$, where $L_{D}$ is the inter-defect distance.

Hydrogen evolution activity measurements. In this work, all potential values are referred to RHE scale. All activity measurements were conducted in a glass electrochemical cell similar to the one used in ref. ${ }^{60}$, except for the measurements of the bare glassy carbon substrates which are performed in a home-made electrochemical cell summarized in ref. ${ }^{57}$ Prior to each measurement, we cleaned the cell with Caro's acid [a 1:3 mixture of $\mathrm{H}_{2} \mathrm{O}_{2}$ (30\% Suprapur, Merck Germany)] and $\mathrm{H}_{2} \mathrm{SO}_{4}(96 \%$ Suprapur, Merck Germany), and rinsed it with ultrapure deionized water (18.2 $\mathrm{M} \Omega \mathrm{cm}$, Evoqua). The reference electrode was a mercury-mercury sulfate electrode (SI Analytics, Germany) in a Luggin capillary filled with $0.1 \mathrm{M} \mathrm{HClO}_{4}\left(70 \%\right.$ Suprapur, Merck Germany) with $E_{\mathrm{Hg} / \mathrm{Hgs}}=-0.71 \mathrm{~V}$ vs RHE. The Luggin capillary is connected to the main cell through a salt bridge. We used a VSP-300 potentiostat (Bio-Logic) for potential control. The activity measurements were conducted in $\mathrm{H}_{2}$-saturated (5.0, Westfalen AG) $0.1 \mathrm{M} \mathrm{HClO}_{4}$ using the hanging meniscus configuration described in ref. ${ }^{61}$ Additionally, in the case of the bead-type $\mathrm{Au}(111)$ as the substrate, the sample was rotated at $1600 \mathrm{rpm}$ using a rotating disk electrode (RDE) system (rotating electrode, Pine). For the HER activity measurement of the bare glassy carbon substrates, we used $\mathrm{N}_{2}$-saturated (evaporated from the liquid nitrogen phase) $1 \mathrm{M} \mathrm{H}_{2} \mathrm{SO}_{4}\left(\geq 96 \%\right.$ VLSI Selectipur $\left.{ }^{\oplus}, \mathrm{BASF}\right)$ as electrolyte and a potassium chloride (puriss. p.a., $\geq 99.5 \%$, Sigma-Aldrich) saturated silver-silver chloride reference electrode (Sigma Aldrich, Germany) with $E_{\mathrm{Ag} / \mathrm{AgCl}}=0.20 \mathrm{~V}$ vs RHE. For the RDE measurements, the counter electrode was the Pt wire in a glass compartment preventing possible contamination of the working solution from dissolved $\mathrm{Pt}$ and subsequent re-deposition into the surface of working electrode.

Electrochemical scanning tunneling microscope. The STM and ECSTM measurements were performed using a Multimode (EC-) STM/(EC-) AFM instrument (Veeco Instrument) equipped with a Nanoscope Universal bipotentiostat and a Nanoscope IIID controller (Nanoscope 5.31r1 software). We used WSXM 5.0 Develop 8.0 software $^{62}$ for microscopic image analysis.

The STM and ECSTM tips were prepared from a Pt/lr alloy wire $(\varnothing=\mathrm{m}$ $0.25 \mathrm{~mm}, \mathrm{Pt} 80 / \mathrm{lr} 20$, GoodFellow) following the procedure described in ref. ${ }^{63}$ The prepared tips were tested by performing normal STM and ECSTM measurements and a typical STM head offset current less than $60 \mathrm{pA}$ should be obtained. The reference and counter electrodes are two Pt wires $(\varnothing=0.5 \mathrm{~mm}$, MaTecK) cleaned with a flame-annealing method and thoroughly rinsed with ultrapure deionized water before each measurement. All electrodes including the TMD samples (working electrode) were positioned in a Teflon holder (ECSTM cell) filled with air-saturated $0.1 \mathrm{M}$ $\mathrm{HClO}_{4}$ electrolyte. To determine the potentials for the case of "HER on" and "HER off", we took cyclic voltammograms for the TMD samples prior to ECSTM imaging.

We note that there is a difference in the applied potential values in the LSV (Fig. 2) and the ECSTM measurements (Fig. 5). Generally, it is not that straightforward to correlate the potentials in the LSV measurements and the HER off/on potentials used in the ECSTM measurements. The LSV measurements were conducted in a well-designed three-electrode cell. The electrolyte and gas-atmosphere were well-controlled during all LSV measurements. However, for the ECSTM measurements, no gasatmosphere control was possible. In particular, the electrolyte is directly exposed to the air, and the ECSTM cell is much smaller than a normal electrochemical cell. In turn, a quasi-reference electrode was used. The reference electrode potential was strongly influenced by the gasatmosphere and the properties (e.g., $\mathrm{pH}$, concentration) of the electrolyte. The potential for HER off/on was determined by the CV recorded before each ECSTM measurement in the same cell under the same atmosphere 
and electrolyte conditions. The LSV measurements were conducted to verify the activity enhancement owing to the helium ion treatment.

The constraint of the ECSTM-method is the tunneling current itself. For instance, the reason for the material selection for the experiments of helium ion treated samples is the fact that the sheet conductivity of $\mathrm{MoSe}_{2}$ is lower compared to $\mathrm{MoS}_{2}$. The lower sheet conductivity of $\mathrm{MoSe}_{2}$ results in a lower tunneling current at identical distance and potential bias between the tip and the sample making the ECSTM measurements on $\mathrm{MoSe}_{2}$ more demanding.

$X$-ray photoelectron spectroscopy. X-ray photoelectron spectroscopy (XPS) measurements were performed with an in-house built UHV setup equipped with a hemispherical energy analyzer PHOIBOS 225 (Specs $\mathrm{GmbH}$; Berlin, Germany). The dual (Mg and Al) X-ray tube anode was operated at an emission current of $20 \mathrm{~mA}$ with a voltage of $12.5 \mathrm{kV}$ at a base pressure of $\leq 8 \times 10^{-9} \mathrm{mbar}$ with non-monochromatic Al $K_{\alpha}$ radiation $(1486.6 \mathrm{eV})$. The data were recorded under normal emission and in medium area mode, corresponding to a circular acceptance area of $2 \mathrm{~mm}$ in diameter. Spectra were recorded with SpecsLab (SPECS Surface Nano Analysis $\mathrm{GmbH}$, ver. 2.85), processed with CasaXPS (Casa Software Ltd, ver. 2.3.17) and further charge corrected by shifting the maximum of the Au $4 \mathrm{f}$ peak to the literature value of $84.0 \mathrm{eV}$ ref. ${ }^{64}$ (found at $83.9 \mathrm{eV}$ ) and by shifting the $\mathrm{C} 1 \mathrm{~s} \mathrm{C}-\mathrm{C}-\mathrm{H} / \mathrm{C}-\mathrm{C}-\mathrm{C}$-component of the adventitious carbon found on the $\mathrm{MoS}_{2}$ bulk single crystal to $284.8 \mathrm{eV}$ ref. ${ }^{65}$ (found at $283.8 \mathrm{eV}$ ). Due to experimental limitations all binding energies obtained in this study are precise to within $\pm 0.2 \mathrm{eV}$. For data processing a Shirley background subtraction and fitting with quasi-Voigt functions (Gaussian-Lorentzian convolutions-ratio of 70 to $30 \%$ (GL (30)) for all transitions beside the $\mathrm{Au}$ $4 f$, for which a $G L(50)$ was used) were performed. The individual element signal intensity was further normalized over the total number of counts by taking the respective photoionization cross-section values from the SPECS database into account ref. ${ }^{66}$

\section{DATA AVAILABILITY}

The data that support the findings of this study are available from the corresponding author upon reasonable request.

\section{ACKNOWLEDGEMENTS}

We acknowledge financial support of the Deutsche Forschungsgemeinschaft (DFG, German Research Foundation) via project BA 5795/3-1 and under Germany's Excellence Strategy-EXC 2089/1-390776260 and the cluster of excellence Nanosystems Initiative Munich (NIM).

\section{AUTHOR CONTRIBUTIONS}

E.M., Y.L., M.G., D.M., J.B. and C.C. conducted the experiments and performed the data analysis. A.H., U.W. and A.S.B. developed the basic ideas and supervised the experimental work. All the authors participated in the discussion of the results and writing the manuscript.

\section{ADDITIONAL INFORMATION}

Supplementary information accompanies the paper on the npj 2D Materials and Applications website (https://doi.org/10.1038/s41699-019-0107-5).

Competing interests: The authors declare no competing interests.

Publisher's note: Springer Nature remains neutral with regard to jurisdictional claims in published maps and institutional affiliations.

\section{REFERENCES}

1. Solomon, S., Plattner, G.-K., Knutti, R. \& Friedlingstein, P. Irreversible climate change due to carbon dioxide emissions. Proc. Natl Acad. Sci. USA 106, 1704-1709 (2009).

2. Chu, S. \& Majumdar, A. Opportunities and challenges for a sustainable energy future. Nature 488, 294-303 (2012).

3. Kibler, L. A. Hydrogen electrocatalysis. ChemPhysChem 7, 985-991 (2006).

4. Wang, M., Wang, Z., Gong, X. \& Guo, Z. The intensification technologies to water electrolysis for hydrogen production - a review. Renew. Sustain. Energy Rev. 29, 573-588 (2014).
5. Edwards, P., Kuznetsov, V., David, W. \& Brandon, N. Hydrogen and fuel cells: towards a sustainable energy future. Energy Policy 36, 4356-4362 (2008).

6. Momirlan, M. \& Veziroglu, T. The properties of hydrogen as fuel tomorrow in sustainable energy system for a cleaner planet. Int. J. Hydrog. Energy 30, 795-802 (2005).

7. Carmo, M., Fritz, D. L., Mergel, J. \& Stolten, D. A comprehensive review on PEM water electrolysis. Int. J. Hydrog. Energy 38, 4901-4934 (2013).

8. Benck, J. D., Chen, Z., Kuritzky, L. Y., Forman, A. J. \& Jaramillo, T. F. Amorphous molybdenum sulfide catalysts for electrochemical hydrogen production: insights into the origin of their catalytic activity. ACS Catal. 2, 1916-1923 (2012).

9. Funke, S. et al. Imaging spectroscopic ellipsometry of $\mathrm{MoS}_{2}$. J. Phys.: Condens. Matter 28, 385301 (2016).

10. Wurstbauer, U., Miller, B., Parzinger, E. \& Holleitner, A. W. Light-matter interaction in transition metal dichalcogenides and their heterostructures. J. Phys. D: Appl. Phys. 50, 173001 (2017).

11. Splendiani, A. et al. Emerging photoluminescence in monolayer $\operatorname{MoS}_{2}$. Nano Lett. 10, 1271-1275 (2010).

12. Liao, J., Sa, B., Zhou, J., Ahuja, R. \& Sun, Z. Design of high-efficiency visible-light photocatalysts for water splitting: $\mathrm{MoS}_{2} / \mathrm{AIN}(\mathrm{GaN})$ heterostructures. J. Phys. Chem. C. 118, 17594-17599 (2014).

13. Parzinger, E. et al. Hydrogen evolution activity of individual mono-, bi-, and fewlayer $\mathrm{MoS}_{2}$ towards photocatalysis. Appl. Mater. Today 8, 132-140 (2017).

14. Chen, Z. et al. Core-shell $\mathrm{MoO}_{3}-\mathrm{MoS}_{2}$ nanowires for hydrogen evolution: a functional design for electrocatalytic materials. Nano Lett. 11, 4168-4175 (2011).

15. Kong, D. et al. Synthesis of $\mathrm{MoS}_{2}$ and $\mathrm{MoSe}_{2}$ films with vertically aligned layers. Nano Lett. 13, 1341-1347 (2013).

16. Benck, J. D., Hellstern, T. R., Kibsgaard, J., Chakthranont, P. \& Jaramillo, T. F. Catalyzing the hydrogen evolution reaction (HER) with molybdenum sulfide nanomaterials. ACS Catal. 4, 3957 (2014).

17. Lin, Z. et al. Defect engineering of two-dimensional transition metal dichalcogenides. 2D Mater. 3, 22002 (2016).

18. Zhang, H. \& Lv, R. Defect engineering of two-dimensional materials for efficient electrocatalysis. J. Mater. 4, 95 (2018).

19. Jaramillo, T. F. et al. Identification of active edge sites for electrochemical $\mathrm{H}_{2}$ evolution from $\mathrm{MoS}_{2}$ nanocrystals. Science 317, 100 (2007).

20. Li, Y. et al. $\mathrm{MOS}_{2}$ nanoparticles grown on graphene: an advanced catalyst for the hydrogen evolution reaction. J. Am. Chem. Soc. 133, 7296 (2011).

21. Lukowski, M. A. et al. Enhanced hydrogen evolution catalysis from chemically exfoliated metallic $\mathrm{MoS}_{2}$ nanosheets. J. Am. Chem. Soc. 135, 10274 (2013).

22. Yin, Y. et al. Contributions of phase, sulfur vacancies, and edges tot he hydrogen evolution reaction catalytic activity of porous molybdenum disulfide nanosheets. J. Am. Chem. Soc. 138, 7965 (2016).

23. Xie, J. et al. Defect-Rich $\mathrm{MoS}_{2}$ ultrathin nanosheets with additional active edge sites for enhanced electrocatalytic hydrogen evolution. Adv. Mater. 25, 5807 (2013).

24. Yu, Y. et al. Layer-dependent electrocatalysis of $\mathrm{MoS}_{2}$ for hydrogen evolution. Nano Lett. 14, 553 (2014).

25. Luxa, J. et al. Tuning of electrocatalytic properties of $\mathrm{MoS}_{2}$ by chalcogenide ion implantation. Appl. Mater. Today 14, 216 (2019).

26. Parzinger, E. et al. Photocatalytic stability of single- and few-layer $\mathrm{MoS}_{2}$. ACS Nano 9, 11302 (2015).

27. Zhang, J. et al. Unveiling active sites for the hydrogen evolution reaction on monolayer $\mathrm{MoS}_{2}$. Adv. Mater. 29, 1701955 (2017).

28. Li, G. et al. All the catalytic active sites of $\mathrm{MoS}_{2}$ for hydrogen evolution. J. Am. Chem. Soc. 138, 16632 (2016).

29. $\mathrm{Li}, \mathrm{H}$. et al. Activating and optimizing $\mathrm{MoS}_{2}$ basal planes for hydrogen evolution through the formation of strained Sulphur vacancies. Nat. Mater. 15, 48 (2016).

30. $\mathrm{Ye}, \mathrm{G}$. et al. Defects engineered monolayer $\mathrm{MoS}_{2}$ for improved hydrogen evolution reaction. Nano Lett. 16, 1097 (2016).

31. Tsai, C. et al. Electrochemical generaton of sulfur vacancies in the basal plane of $\mathrm{MoS}_{2}$ for hydrogen evolution. Nat. Commun. 8, 15113 (2017).

32. Meng, C., Lin, M., Du, X. \& Zhou, Y. Molybdenum disulfide modified by laser irradiation for catalyzing hydrogen evolution. ACS Sustain. Chem. Eng. 7, 6999 (2019).

33. Luxa, J. et al. Tuning of electrocatalytic properties of $\mathrm{MoS}_{2}$ by chalcogenide ion implantation. Appl. Mater. Today 14, 216 (2019).

34. Castellanos-Gomez, A. Why all the fuss about 2D semiconductors? Nat. Photonics 10, 202-204 (2016).

35. Liu, Y. et al. Van der Waals heterostructures and devices. Nat. Rev. Mater. 1, 16042 (2016).

36. Klein, J. et al. Impact of intrinsic and extrinsic disorder on the electronic and optical properties of $\mathrm{MoS}_{2}$ arxiv 1905.01242, 2019.

37. Vancsó, P. et al. The intrinsic defect structure of exfoliated $\mathrm{MoS}_{2}$ single layers revealed by Scanning Tunneling Microscopy. Sci. Rep. 6, 29726 (2016).

38. Hong, J. et al. Exploring atomic defects in molybdenum disulphide monolayers. Nat. Commun. 6, 6293 (2015). 
39. Komsa, H.-P. \& Krasheninnikov, A. V. Native defects in bulk and monolayer $\mathrm{MoS}_{2}$ from first principles. Phys. Rev. B 91, 125304 (2015).

40. Tsai, C., Chan, K., Abild-Pedersen, F. \& Nørskov, J. K. Active edge sites in $\mathrm{MoSe}_{2}$ and $\mathrm{WSe}_{2}$ catalysts for the hydrogen evolution reaction: a density functional study. Phys. Chem. Chem. Phys. 16, 13156 (2014).

41. Yoshimoto, S., Kim, Y.-G., Sato, K., Inukai, J. \& Itaya, K. Potential-induced phase transition of low-index Au single crystal surfaces in propylene carbonate solution. Phys. Chem. Chem. Phys. 14, 2286 (2012).

42. Lu, Q., Yu, Y., Ma, Q., Chen, B. \& Zhang, H. 2D transition-metal-dichalcogenidenanosheet-based composites for photocatalytic and electrocatalytic hydrogen evolution reactions. Adv. Mater. 28, 1917-1933 (2016).

43. Pfisterer, J. H. K., Liang, Y., Schneider, O. \& Bandarenka, A. S. Direct instrumental identification of catalytically active surface sites. Nature 549, 74-77 (2017).

44. Li, G. et al. All The Catalytic Active Sites of $\mathrm{MoS}_{2}$ for hydrogen evolution. J. Am. Chem. Soc. 138, 16632-16638 (2016).

45. Benck, J. D., Hellstern, T. R., Kibsgaard, J., Chakthranont, P. \& Jaramillo, T. F. Catalyzing the Hydrogen Evolution Reaction (HER) with Molybdenum Sulfide Nanomaterials. ACS Catal. 4, 3957-3971 (2014).

46. Tsai, $C$. et al. Electrochemical generation of sulfur vacancies in the basal plane of $\mathrm{MoS}_{2}$ for hydrogen evolution. Nat. Commun. 8, 15113 (2017).

47. Ouyang, Y. et al. Activating inert basal planes of $\mathrm{MoS}_{2}$ for hydrogen evolution reaction through the formation of different intrinsic defects. Chem. Mater. 28, 4390-4396 (2016).

48. Surrente, A. et al. Defect healing and charge transfer-mediated valley polarization in $\mathrm{MoS}_{2} / \mathrm{MoSe}_{2} / \mathrm{MoS}_{2}$ Trilayer van der Waals Heterostructures. Nano Lett. 17, 4130-4136 (2017).

49. Le, D., Rawal, T. B. \& Rahman, T. S. Single-layer $\mathrm{MoS}_{2}$ with sulfur vacancies: structure and catalytic application. J. Phys. Chem. C. 118, 5346-5351 (2014).

50. Lee, J. et al. Hydrogen evolution reaction at anion vacancy of two-dimensional transition-metal dichalcogenides: ab initio computational screening. J. Phys. Chem. Lett. 9, 2049-2055 (2018).

51. Ambrosi, A., Sofer, Z. \& Pumera, M. $2 \mathrm{H} \rightarrow 1 \mathrm{~T}$ phase transition and hydrogen evolution activity of $\mathrm{MoS}_{2}, \mathrm{MoSe}_{2}, \mathrm{WS}_{2}$ and $\mathrm{WSe}_{2}$ strongly depends on the $\mathrm{MX}_{2}$ composition. Chem. Commun. 51, 8450-8453 (2015).

52. Uosaki, K. et al. Highly efficient electrochemical hydrogen evolution reaction at insulating boron nitride nanosheet on inert gold substrate. Sci. Rep. 6, 32217 (2016).

53. $\mathrm{Li}, \mathrm{H}$. et al. Activating and optimizing $\mathrm{MoS}_{2}$ basal planes for hydrogen evolution through the formation of strained sulphur vacancies. Nat. Mater. 15, 48-53 (2016).

54. Tsai, C., Abild-Pedersen, F. \& Nørskov, J. K. Tuning the $\mathrm{MoS}_{2}$ edge-site activity for hydrogen evolution via support interactions. Nano Lett. 14, 1381-1387 (2014)
55. Klein, J. et al. Site-selectively generated photon emitters in monolayer $\mathrm{MoS}_{2}$ via local helium ion irradiation. Nat. Comm 10, 2755 (2019).

56. Castellanos-Gomez, A. et al. Deterministic transfer of two-dimensional materials by all-dry viscoelastic stamping. 2D Mater. 1, 011002 1-8 (2014).

57. Parzinger, E. et al. Hydrogen evolution reaction of individual Mono-, Bi-, and Fewlayer $\mathrm{MoS}_{2}$ towards photocatalysis. Appl. Mater. today 8, 132-140 (2017).

58. Kibler, L. A. Preparation and characterization of noble metal single crystal electrode surfaces. International Society of Electrochemistry, 1-55 (2003).

59. Hlawacek, G., Veligura, V., van Gastel, R. \& Poelsema, B. Helium Ion Microscopy. J. Vac. Sci. Technol. B, Nanotechnol. Microelectron.: Mater., Process., Meas., Phenom. 32, 02081 1-13 (2014).

60. Calle-Vallejo, F. et al. Finding optimal surface sites on heterogeneous catalysts by counting nearest neighbors. Science 350, 185-189 (2015).

61. Colić, V. et al. Experimental aspects in benchmarking of the electrocatalytic activity. ChemElectroChem 2, 143-149 (2015).

62. Horcas, I. et al. WSXM: a software for scanning probe microscopy and a tool for nanotechnology. Rev. Sci. Instrum. 78, 013705 (2007).

63. Pfisterer, J. H. K., Liang, Y., Schneider, O. \& Bandarenka, A. S. Direct instrumental identification of catalytically active surface site. Nature 549, 74-77 (2017).

64. Anthony, M. T. \& Seah, M. P. XPS: Energy calibration of electron spectrometers. 2 Results of an interlaboratory comparison. Surf. Interface Anal. 6, 107-115 (1984).

65. Bettis Homan, S. et al. Ultrafast exciton dissociation and long-lived charge separation in a photovoltaic Pentacene- $\mathrm{MoS}_{2}$ van der Waals heterojunction. Nano Lett. 17, 164-169 (2017).

66. http://www.casaxps.com/, CasaXPS VAMAS Processing Software.

(i) Open Access This article is licensed under a Creative Commons Attribution 4.0 International License, which permits use, sharing, adaptation, distribution and reproduction in any medium or format, as long as you give appropriate credit to the original author(s) and the source, provide a link to the Creative Commons license, and indicate if changes were made. The images or other third party material in this article are included in the article's Creative Commons license, unless indicated otherwise in a credit line to the material. If material is not included in the article's Creative Commons license and your intended use is not permitted by statutory regulation or exceeds the permitted use, you will need to obtain permission directly from the copyright holder. To view a copy of this license, visit http://creativecommons. org/licenses/by/4.0/.

(c) The Author(s) 2019 